\title{
1 Event Localization in Bulk Scintillator Crystals Using Coded Apertures
}

2 K.P. Ziock ${ }^{\mathrm{a}, \mathrm{b}, *}$, J.B. Braverman ${ }^{\mathrm{b}}$, L. Fabris ${ }^{\mathrm{a}}$, M.J. Harrison ${ }^{\mathrm{a}}$, D. Hornback ${ }^{\mathrm{a}}$, J. Newby ${ }^{\mathrm{a}}$

$3 \quad$ a Oak Ridge National Laboratory, Oak Ridge, TN USA

$4 \quad{ }^{\mathrm{b}}$ Department of Physics and Astronomy, University of Tennessee, Knoxville, TN, USA

5 Keywords: Scintillation Detector, Position-Sensitive Detector, Coded Aperture Imaging, Gam-

6 ma-ray Detector

\section{ABSTRACT}

8 The localization of radiation interactions in bulk scintillators is generally limited by the size of

9 the light distribution at the readout surface of the crystal/light-pipe system. By finding the cen-

10 troid of the light spot, which is typically of order centimeters across, practical single-event local-

11 ization is limited to $\sim 2 \mathrm{~mm} / \mathrm{cm}$ of crystal thickness. Similar resolution can also be achieved for

12 the depth of interaction by measuring the size of the light spot. Through the use of near-field

13 coded-aperture techniques applied to the scintillation light, light transport simulations show that

14 for 3-cm-thick crystals, more than a five-fold improvement (millimeter spatial resolution) can be

15 achieved both laterally and in event depth. At the core of the technique is the requirement to re-

16 solve the shadow from an optical mask placed in the scintillation light path between the crystal

17 and the readout. In this paper, experimental results are presented that demonstrate the overall

18 concept using a 1D shadow mask, a thin-scintillator crystal and a light pipe of varying thickness

19 to emulate a 2.2-cm-thick crystal. Spatial resolutions of $\sim 1 \mathrm{~mm}$ in both depth and transverse to

20 the readout face are obtained over most of the crystal depth.

\section{INTRODUCTION}

22 Gamma-ray detectors based on inorganic scintillator crystals are widely used throughout the

23 radiation detection community. Their useful balance of energy resolution, stopping power, and

24 cost per unit volume has made them essential to a broad range of fields such as fundamental 
25 physics, high-energy astrophysics, homeland security, and nuclear medicine. In many of these

26 applications, one is interested in both the energy of the gamma radiation and its interaction loca-

27 tion within the detector. Several approaches have been developed to measure the latter. The most

28 straightforward approach is to place a series of readout devices on one or multiple surfaces of a

29 bulk crystal and look at how the light is shared between them. The location of each interaction is

30 estimated using some form of centroiding algorithm with the spatial resolution limited by the

31 size of the light spot at the readout surface divided by the square root of the number of detected

32 scintillation photons. Anger cameras [1] are the primary example of this approach, and they can

33 achieve a spatial resolution of a few millimeters along the instrumented face [2]. The problem

34 with the centroiding approach is that the size of the light spot is of order the distance that the

35 event occurs from the readout surface, and so the ability to locate the event is limited $(\sim 2 \mathrm{~mm} / \mathrm{cm}$

36 of crystal thickness at $500 \mathrm{keV})$.

37 One means to improve spatial resolution is to subdivide the crystal to restrict the spread of

38 the light to a single small "crystalet." Using this approach, one can achieve better spatial resolu-

39 tion $(<1 \mathrm{~mm})$. However, if the crystalets are too small, then they will "leak" energy to neighbor-

40 ing locations due to finite electron ranges, K-escape peaks, and Compton scatter. Further, to pro-

41 vide good stopping power and maintain good spatial resolution requires crystalets with a high

42 aspect ratio. This tends to limit energy resolution since fewer of the scintillation photons created

43 in events far from the readout surface are detected.

$44 \quad$ When one requires the location of an event in all three dimensions, the problem becomes

45 even harder. In detectors that use bulk crystals, one can determine the depth of an event by

46 measuring the size of the light spot at the readout. This provides a resolution similar to that of an

47 event's lateral location. In segmented systems with high aspect crystalets, one can instrument 
48 both ends of the crystalet and use light sharing (or even light arrival time for larger bar pixels).

49 The thickness of such an assembly will be limited by the acceptable light loss from multiple re-

50 flections along the crystalet. In small systems $\left(\sim 1 \mathrm{~cm}^{3}\right)$, sub-millimeter spatial resolution can be

51 achieved, but with a significant increase in the system complexity [3].

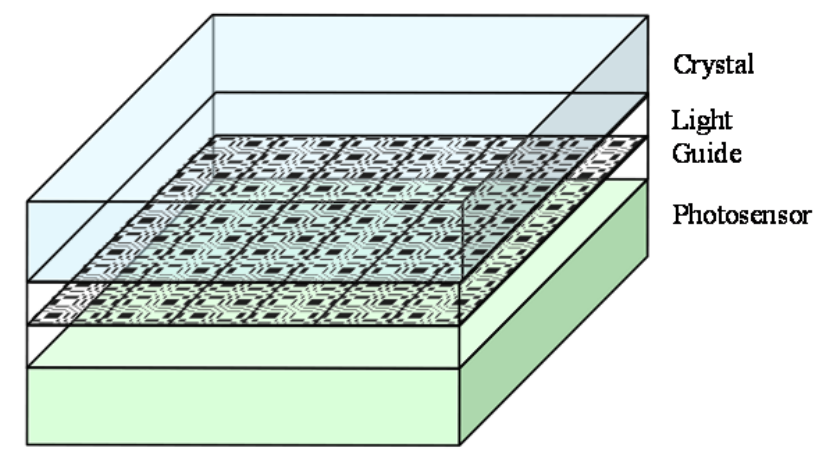

Fig. 1. Detector concept. An optical coded-aperture shadow mask is positioned in the light pipe connecting a bulk scintillator crystal with a position-sensitive photosensor.

We report the first experimental results of a new approach that promises to provide the spa-

54 tial resolution achievable with a pixelated system in a bulk scintillator. The technique is shown

55 schematically in Fig. 1. It uses a coded-aperture shadow mask [4] in the light pipe that connects

56 the scintillator crystal to a position-sensitive readout device. The shadow mask encodes the event

57 location in all three dimensions, allowing one to locate events within the crystal. For the location

58 parallel to the readout surface, standard coded-aperture cross-correlation calculations are used

59 [4]. To determine the depth of an event, the approach takes advantage of the fact that the mask

60 pattern projected on the readout device is magnified, with the amount of magnification deter-

61 mined by the depth of the event within the crystal. By sequentially reconstructing an event at dif-

62 ferent depths (magnifications), one can a posteriori "vary the focus" of the system and look for

63 the sharpest image. Extensive light-transport simulations have been conducted and indicate that

64 voxel sizes of order a cubic millimeter can be achieved at energies as low as several hundred 
$65 \mathrm{keV}$ in 3-cm-thick scintillators [5,6]. For the first measurements reported here, a 1D coded aper-

66 ture was used to provide 2D event reconstructions - depth and one dimension lateral to the

67 readout surface.

68

69

70

71

72

73

74

75

76

77

78

79

80

81

82

83

\section{EXPERIMENTAL DESIGN}

A photograph and schematic of the hardware used for the experiment are shown in Fig. 2. In the experiment a thin scintillator crystal [1-mm-thick $\mathrm{NaI}(\mathrm{Tl})$, index of refraction $\mathrm{n}=1.85$ ] is mounted to a quartz light pipe $(\mathrm{n}=1.458)$ that contains the shadow mask. Additional light pipe below the mask connects to the readout. Changing the amount of light pipe between the crystal and the mask provides a means of emulating a thick crystal with events located at different known depths.
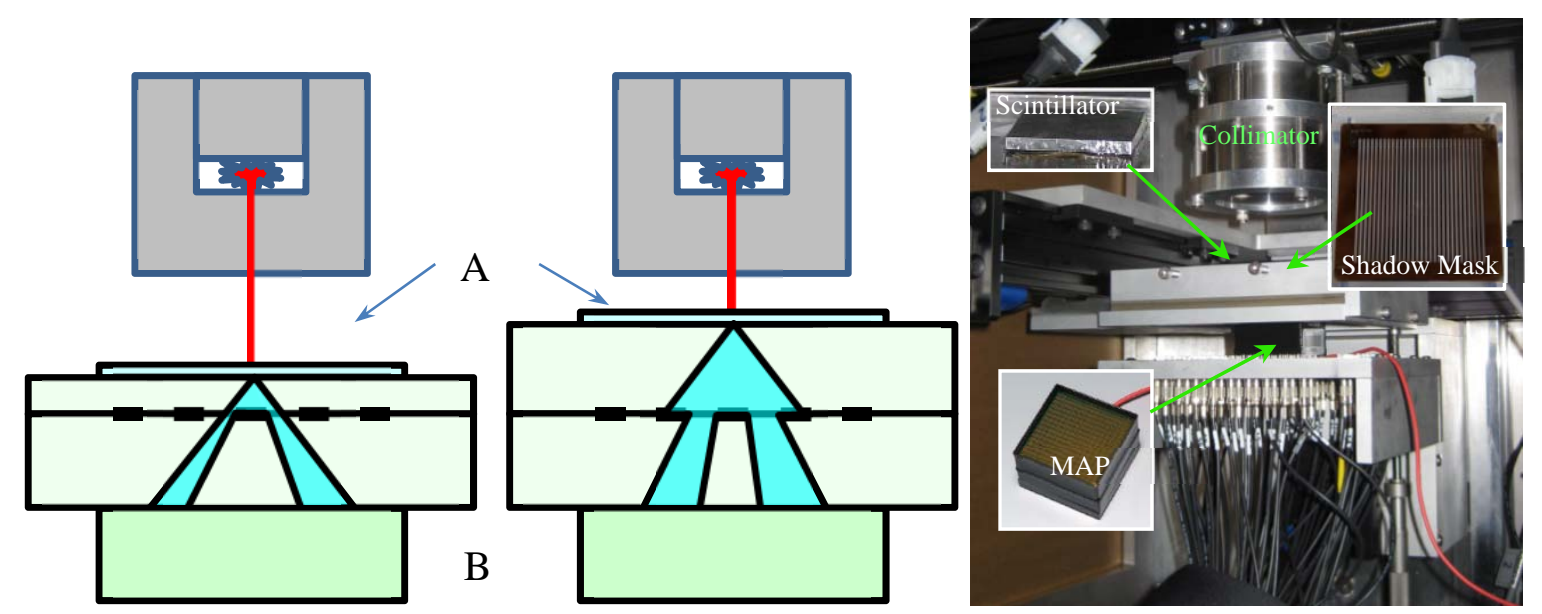

Fig. 2. Schematics of the experimental layout (left, center). A thin crystal (A) is placed on top of the light pipe/mask assembly. Scintillation light is detected by the MAP (B). The amount of light pipe between the mask and the crystal can be varied to emulate events at different locations within a thick crystal. Events at the bottom (left) generate a mask shadow with a greater magnification at the MAP compared to an event at the top of the crystal. The experimental hardware is shown on the right.

The experiment used three Hamamatsu 9500 multi-anode photomultiplier (MAP) tubes [7] arranged side by side. All of the anodes perpendicular to the three-tube axis were connected to form readout strips. This MAP array was used below a shadow mask based on multiple repeti- 
84 tions of a rank-7, 1D modified uniformly redundant array (MURA) coded aperture [8]. To local-

85 ize the radiation to a given region of the crystal, a tungsten collimator was used. This provided a

86 beam $\sim 1.2 \mathrm{~mm}$ in diameter at the crystal surface. Connections between optical elements were

87 made using optical coupling compound $(\mathrm{n}=1.465)$ [9].

\subsection{CRYSTAL THICKNESS}

The maximum displacement of the thin crystal in the experiments was $14.7 \mathrm{~mm}$. Because

90 the angular divergence of the light cloud increases when transitioning into the quartz (index of

91 refraction $\sim 1.5$ ) from the higher index scintillator ( 1.8), a smaller thickness of quartz generates

92 the same spread as a greater thickness of scintillator crystal. To obtain the conversion factor be-

93 tween event depths in a bulk scintillator and the thin-crystal-quartz-light-pipe analog, we ran

94 light transport simulations using GEANT4 [10] similar to those reported earlier [5, 6]. In the

95 simulations, a scintillation event was modelled by launching 40,000 photons at the top of the ap-

96 paratus shown in Fig. 2. The photons were launched randomly in all directions. (Note that the top

97 and sides of the optical system are painted black, and any photons hitting those surfaces were

98 removed from the transport simulations.) The locations where photons reached the photocathode

99 of the MAPs were recorded and mapped to the anode structure used in the experiment. These

100 events were then imaged as described in Section 3.1.3 below to determine the event depth. For

101 systems with different optical thicknesses, two configurations were run, one with a thin $\mathrm{NaI}(\mathrm{Tl})$

102 crystal with a quartz light pipe, and one where the light pipe was replaced by a material with the

103 same refractive index as that of NaI. The results are plotted in Fig. 3 and show a linear relation-

104 ship with a slope of 0.63 . This factor is used in Table 1 to relate the real geometry to the emulat-

105 ed event location within a thick crystal. It indicates that the 15-mm-thick light pipe is comparable 106 to a 22.5-mm-thick crystal. 


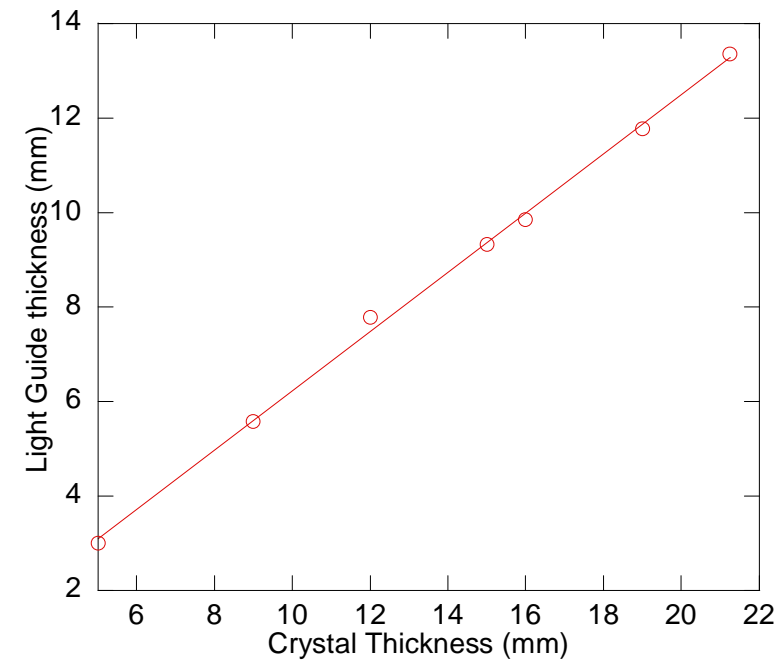

Fig. 3. Relationship between the thickness of the light pipe emulation and a bulk crystal.

\subsection{READOUT SYSTEM}

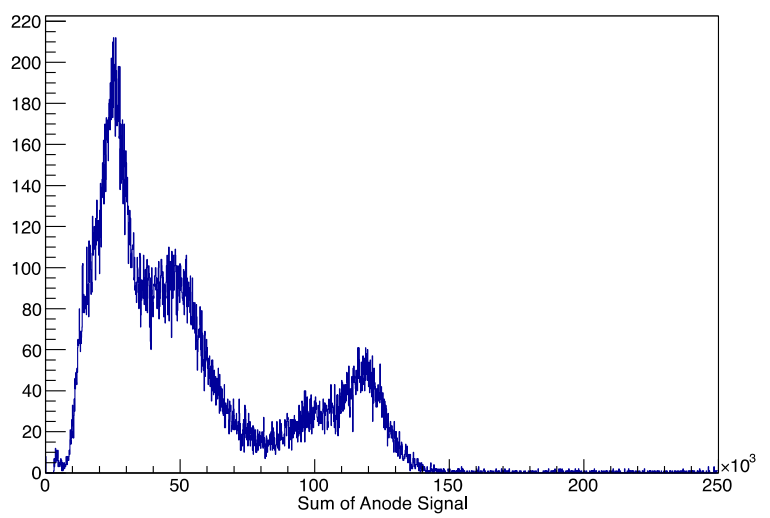

Fig. 4 Spectrum obtained from a ${ }^{133}$ Ba source using the peaklet integration approach .

112 The design of the experiment was dominated by the 64 channels of readout available to perform

113 the work. The data acquisition system comprised four, $250 \mathrm{MHz}$ digitizers [11] in a VME crate

114 read out using a personal computer. Due to noise and signal reflection issues when the digitizers

115 were configured to run in a high-input impedance mode, the $50 \Omega$ mode was used. To drive this

116 the output of each row of anodes was sent through a Phillips 776, times 10 amplifier [12] before

117 being passed to the digitizers. Residual baseline noise around $1 \mathrm{MHz}$ meant that simply integrat-

118 ing the total charge received during the decay period of the scintillator light (a 3200-ns integra- 
119 tion window was used) yielded poor energy resolution. To overcome this a modified peaklet

120 analysis procedure was implemented. After a trigger was received (based on the signal from the

121 last dynode of the center MAP), the 3200-ns-wide inspection window was opened for each chan-

122 nel. The signal recorded during the window was searched for the many small (single or a few)

123 photoelectron pulses (peaklets) that arrived in each channel. The signal was integrated for those

124 times that the signal level was above an empirically determined threshold that was set to avoid

125 triggering on noise. With the threshold set high enough to avoid noise, some charge was lost in

126 the bins immediately before and just after the signal rose above or fell below the threshold level.

127 The integration routine was modified to explicitly include data from those time bins. This tech-

128 nique provided good energy resolution and was developed during initial tests with a single MAP

129 to show that single photoelectrons could be detected. A sample energy spectrum of ${ }^{133} \mathrm{Ba}$ ob-

130 tained by the system without a shadow mask in the light pipe is shown in Fig. 4. As expected,

131 addition of the mask reduced the peak location due to the light blocked by the mask by a factor

132 of two and worsened the energy resolution for the $356-\mathrm{keV}$ peak from $14 \%$ full width at half

133 maximum (FWHM) to 20\% ( root-two worse). These are reasonable values given that the crys-

134 tal sides are polished and the top and sides of the crystal are painted black to reduce unwanted

135 reflections from those surfaces that would make analysis of the shadow data more difficult.

$136 \quad 2.3$ MASK DESIGN

137 Optical masks with $2 \mu \mathrm{m}$ of chromium on 6.35-mm-thick quartz blanks were purchased

138 from a vendor [13] that supplies photomasks to the semiconductor industry. Specifications from

139 the manufacturer indicate residual transmission at $410 \mathrm{~nm}$ of only $0.032 \%$. One side of the masks

140 is shiny, and this surface was oriented away from the crystal. A rank-7 MURA coded aperture

141 [8] was chosen with a pixel pitch of $1.4 \mathrm{~mm}$ (Fig. 5). 
Fig. 5. Photograph of the 1D, rank-7 MURA shadow mask. The double arrow indicates the base period of the MURA. As described in the previous simulation papers [5,6], selection of the coded-aperture pattern is a

145 balance between the overall size of the base pattern and the resolution of the readout device. In 146 short, the mask contains many cycles of the base pattern since one must observe the shadow 147 from a complete pattern in order to obtain good image reconstruction. The light spots from 148 events at the bottom of the crystal (closest to the mask and MAP readout) will have the smallest 149 width at the mask plane, with the size determined by the thickness of light pipe between the bot150 tom of the crystal and the mask. Balancing this criterion (which pushes one toward a smaller 151 base mask pattern size) is the fact that repetition of the base pattern means one must be able to

152 localize an event to a single pattern without using the coded-aperture approach, because the cod153 ed-aperture reconstruction can only localize the event within a pattern. If the event is ascribed to 154 the wrong cycle of the pattern, then it will be incorrectly located in the mask imaging direction 155 by the same integer number of patterns that the event was misplaced in the initial localization 156 (see Section 3 on data analysis). This condition is important for events at the top of the crystal 157 (those that fall the furthest from the mask and MAPs), since these are the ones that will be most 158 diffuse and thus hardest to locate. This tends to drive the design toward a larger base mask pat159 tern size. At the same time, the size of the smallest mask feature projected onto the MAP photo160 cathodes must be large enough to be resolved by the MAP. This impacts the number of pixels 
161 within a given pattern (rank) and the length of the light pipe between the mask and the MAPs.

162 The larger the distance, the more the magnification of the pattern, making it easier to resolve the

163 mask feature size, but also the wider the pattern will be, making it harder to localize the event to

164 the correct mask pattern.

165 The Hamamatsu 9500 MAPs used here have a primary pixel pitch of $3.04 \mathrm{~mm}$. Based on a

166 crystal emulation thickness of $20 \mathrm{~mm}$, and balancing the terms described above, we used a min-

167 imum magnification of 2.14 for an event at the top of the crystal. With a base pattern size at the

168 mask for a 24.85-mm-thick light pipe below the emulated crystal, this implied the use of the

169 rank-7 pattern with a pitch of $1.4 \mathrm{~mm}$. These values correspond to magnification factors of 2.23

170 to 4.82, giving base MURA feature sizes at the MAP varying from 3.12 to $6.75 \mathrm{~mm}$ or 1.02 to

1712.2 pixels, respectively.

172

\section{DATA COLLECTION}

173 Data were collected with the rank-7 photomask described in Section 2.3 above. The overall 174 geometries studied are summarized in Table 1. At least 20,000 events were collected at each

175 depth with the acquisition system triggered by the last dynode signal of the center H9500.

176 The conceptual design of the hardware was meant to allow the amount of light pipe between

177 the mask and the crystal to be changed without changing the alignment between the collimator,

178 the mask, and the MAPs. However, this was not found to work well in practice. In fact, the

179 whole assembly was sometimes rebuilt between measurements with different light pipe thick-

180 nesses. Consequently, the transverse location of the beam varied somewhat between the different

181 measurements. As discussed below, an additional complication was that at times the order of the

182 MAPs was changed, which affected the gains (and quantum efficiency) for the different strips. 
Table 1

Emulated event depths and results.

\begin{tabular}{ccccccc}
\hline $\begin{array}{c}\text { Mask to } \\
\text { crystal } \\
\text { center } \\
(\mathrm{mm})\end{array}$ & $\begin{array}{c}\text { Emulated } \\
\text { event depth in } \\
\text { crystal } \\
(\mathrm{mm})\end{array}$ & $\begin{array}{c}\text { Depth } \\
\text { resolution } \\
\text { FWHM } \\
(\mathrm{mm})\end{array}$ & $\begin{array}{c}\text { In-crystal } \\
\text { depth } \\
\text { resolution } \\
\text { FWHM } \\
(\mathrm{mm})\end{array}$ & $\begin{array}{c}\text { In-crystal depth } \\
\text { resolution, crys- } \\
\text { tal thickness } \\
\text { deconvolved } \\
\text { FWHM } \\
(\mathrm{mm})\end{array}$ & $\begin{array}{c}\text { X-resolution } \\
\text { FWHM } \\
(\mathrm{mm})\end{array}$ & $\begin{array}{c}\text { X resolution } \\
\text { source-width } \\
\text { deconvolved } \\
\text { FWHM } \\
(\mathrm{mm})\end{array}$ \\
\hline 6.5 & 0.0 & 0.65 & 1.03 & $<0.54$ & 1.67 & 1.43 \\
9.0 & 4.0 & 0.65 & 1.03 & $<0.54$ & 1.22 & 0.87 \\
13.0 & 10.3 & 0.65 & 1.03 & $<0.54$ & 1.45 & 1.17 \\
20.7 & 22.5 & 1.67 & 2.65 & 2.50 & 1.16 & 0.78 \\
\hline
\end{tabular}

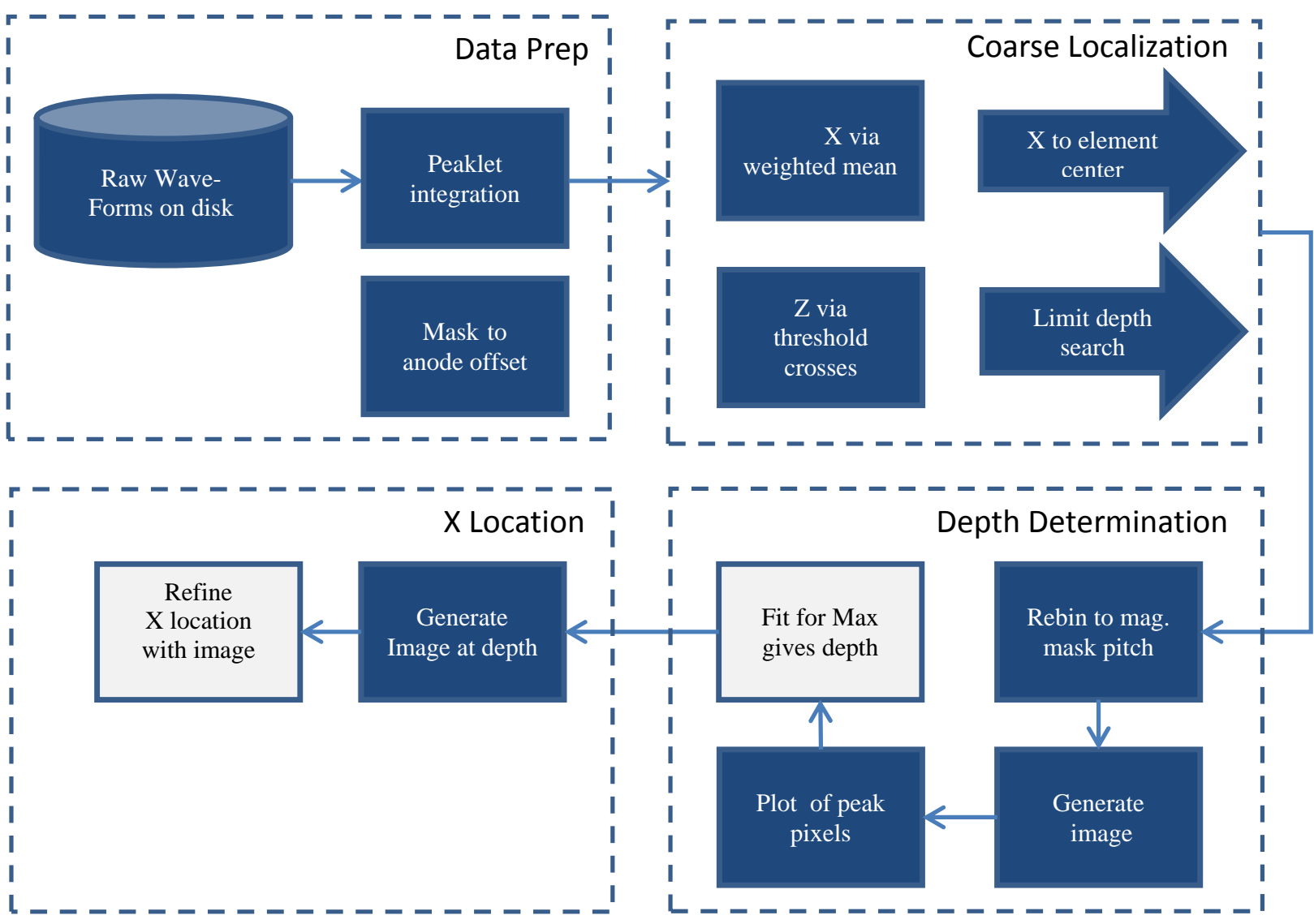

186 Fig. 6. Schematic diagram of the event processing steps.

\subsection{DATA ANALYSIS}

A number of different data processing approaches were explored to optimize the results ob-

189 tained with the system. The final approach used is shown schematically in Fig. 6 and is described

190 in detail below. 


\subsubsection{Preprocessing}

Before individual events could be processed, we had to determine the alignment between the 193 mask pattern and the MAP anodes for each measurement because of alignment shifts when the 194 system was reconfigured. Knowledge of this alignment impacts rebinning the events for depth

195 processing because the MAP anode spacing is not uniform but varies at the edge of the tube and 196 has a gap between tubes. To determine the alignment, all of the events for a given depth were 197 reconstructed using the coded-aperture cross-correlation technique and the known event depth 198 and mask cycle. The average of the x-location obtained was then used as the known offset be199 tween the two patterns (mask and anode) for processing individual events for each emulated 200 depth.

201 Ideally, the data would also have been corrected for spatial variations in the MAP gain and 202 quantum efficiency. Unfortunately, these were not characterized and, worse, the MAP positions 203 were exchanged for some of the configurations. To correct for the most egregious variations that

204 occurred at tube boundaries, an assumption was made that the total light from many events 205 should yield a smooth distribution. When the response for the unprocessed events was plotted as 206 a function of anode strip number, discontinuities at the interface between tubes were sometimes 207 noted and here gain variations were applied to provide a smoothed response.

208 Once the overall gains were set, the individual event data were processed using the peaklet 209 integration technique discussed in Section 2.2. An energy threshold was used to keep only events

210 in the main peak at $356 \mathrm{keV}$ from the ${ }^{133} \mathrm{Ba}$ source used for the measurements. Because of the

211 finite MAP area, the thick light pipe geometries, and the blackened sides of the system, the total 212 light collected varied by $8.5 \%$ between events emulated at the top and bottom of the 2-cm-thick 213 crystal. The energy cut was selected separately for each depth to avoid contamination with the 
214 81-keV line from the source and by lead and tungsten K-Shell x-rays for events with the thinner

215 light pipes that would have occurred with a single wide threshold used for all depths. (Analytical 216 calculations [14] and simulations show this is a much smaller effect if a larger readout area is 217 used.)

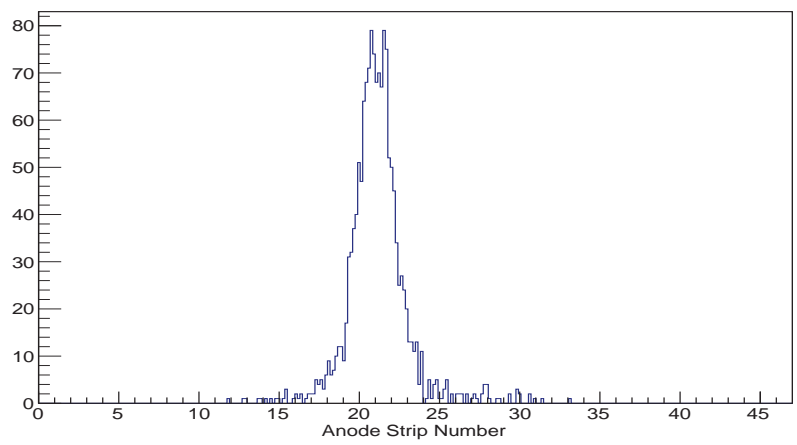

218

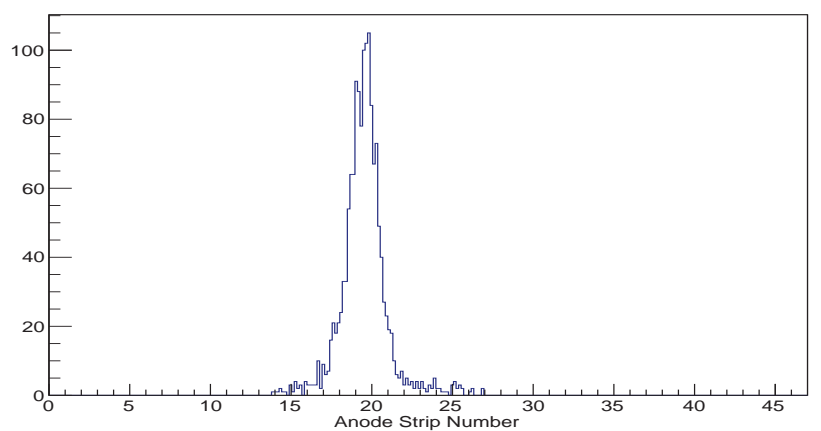

220 Fig. 7. Histograms of event $x$-locations at the bottom (bottom) and top (top) of the emulated crystal found 221 using a weighted mean.

\section{$222 \quad$ 3.1.2 Coarse Event Localization}

223 The first step in processing the individual events was to determine the coarse event location 224 in $\mathrm{x}$ (along the direction of mask modulation) in order to localize the event to a single cycle of 225 the mask pattern. A simple weighted mean was found to give the best results, and sample histo226 grams of event distributions are shown in Fig. 7.

227 With a known coarse x-location, a rough estimate of the depth was obtained by counting the 228 number of times that the ADC counts per channel vs channel numberfor an event exceeded a 
229 threshold set at $40 \%$ of the maximum value for the event. This worked because the broader the

230 light spot at the mask, the more open and closed elements are shadowed and hence the more

231 crossovers occur. The overall distributions of crossovers for events at the top and bottom of the

232 emulated crystal are shown in Fig. 8. The strong peaks at even numbers are due to the fact that if

233 the signal goes over the threshold, it generally also comes back down. The information was used

234 to limit the lowest depths over which the finer depth determination was performed, both to in-

235 crease processing speed and to reduce crosstalk that was found to occur between events at the top 236 and bottom emulated locations.
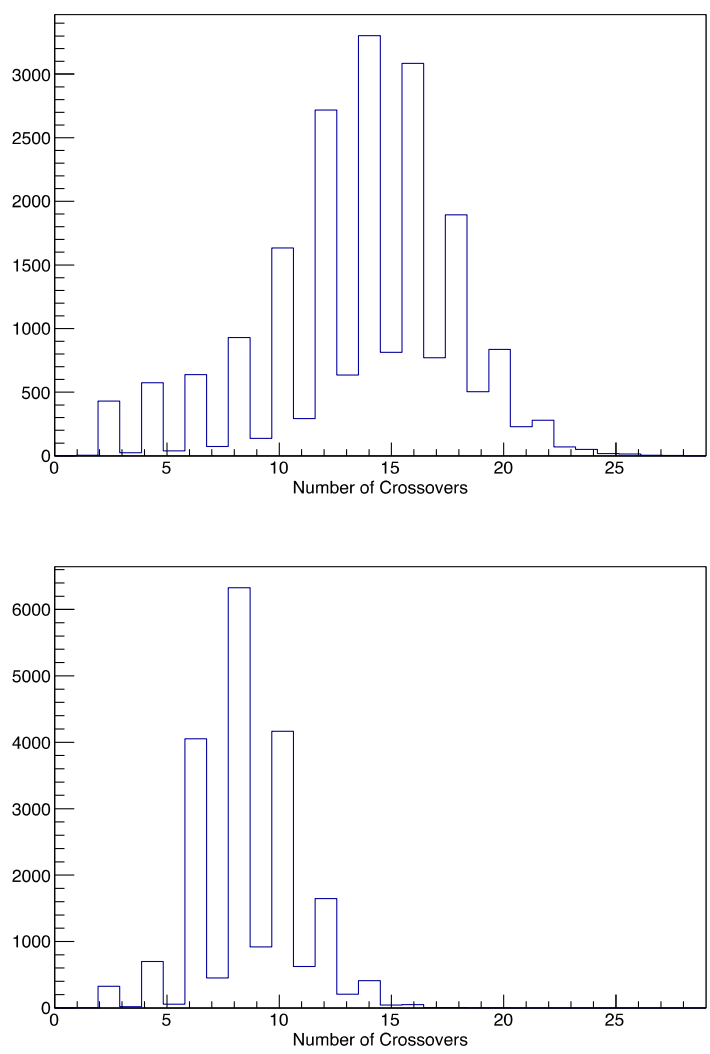

239 Fig. 8. Histograms for the number of crossovers (see text) for all events launched from the top (top) and bot-

240 tom (bottom) of the emulated crystal.

\section{3.1.3 Depth Determination}

242 After the coarse $\mathrm{x}$ and depth locations had been determined, the center of the mask element 
nearest the x-location was chosen as the "starting” event x-location, and precise depth determina-

244 tion was performed. For this, the data were processed for a range of depths by generating a 1D

245 coded-aperture image using the correct magnification and binning for that depth. This amounts to

246 “refocusing” the system a posteriori to obtain the sharpest image. Sample images for a single

247 event at a number of reconstructed depths are given in Fig. 9. As can be seen, as one varies the

248 reconstruction depth, a degraded image progresses to a sharp point-source image, and then de-

249 grades again. A plot of the peak intensity versus reconstruction depth for the event is given in

250 Fig. 10.

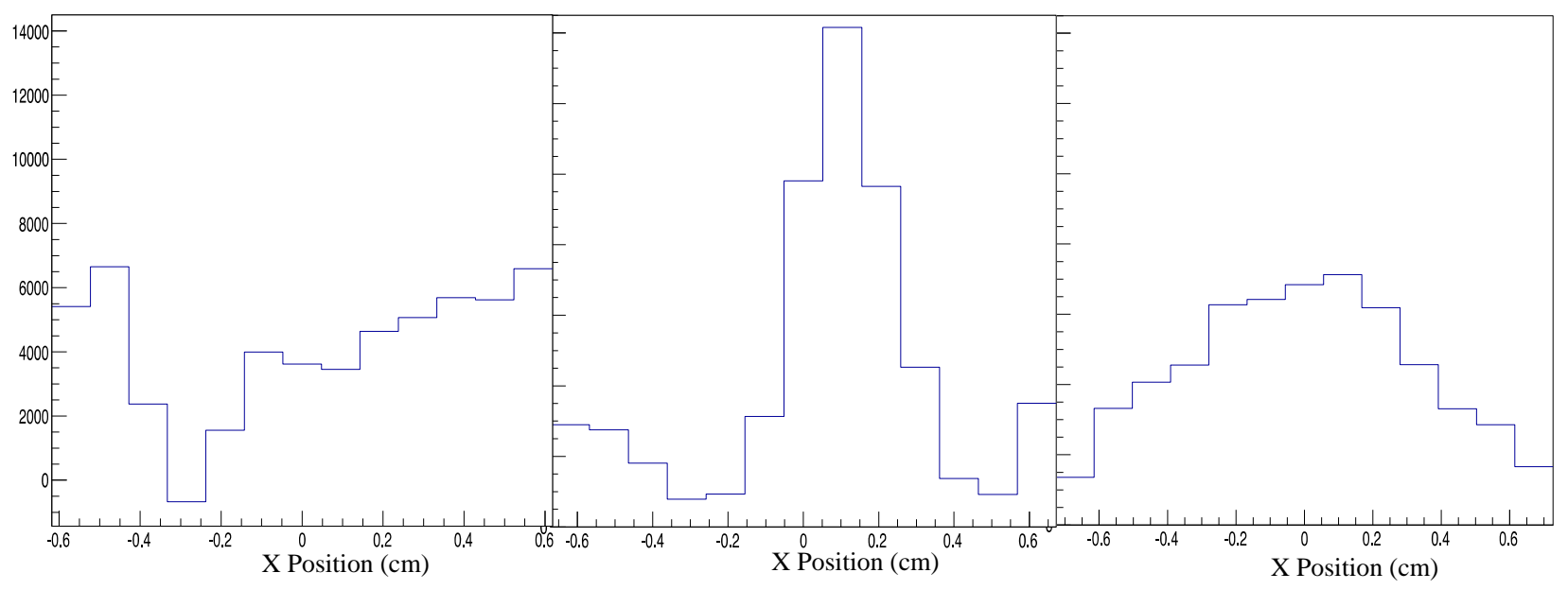

Fig. 9. From left to right, sample reconstructed images obtained using depths of 6.5, 9.2, and $12.0 \mathrm{~mm}$, respec-

253 tively. The peak is sharpest at the correct depth of $9.2 \mathbf{~ m m}$. A plot of peak height vs depth for this event is

254 given in Fig. 10. The y-scale is arbitrary but the same for all plots.

255 To generate the image at each depth, the data were rebinned from the pitch of the anode

256 strips to an idealized “detector” that had a pitch twice that of the magnified mask pattern for that

257 depth, centered on the starting location. A standard cross-correlation reconstruction algorithm

258 was then used [4] to generate the image. Note that the data from all strips were used, with the

259 data from neighboring cycles of the mask folded back to the correct detector element represent-

260 ing the same part of the cycle [15]. To generate the rebinned detector values, the signal levels 
261 were weighted by the actual anode strip geometries, including the gaps between PMTs and the 262 slightly greater width of the edge anodes of the PMTs

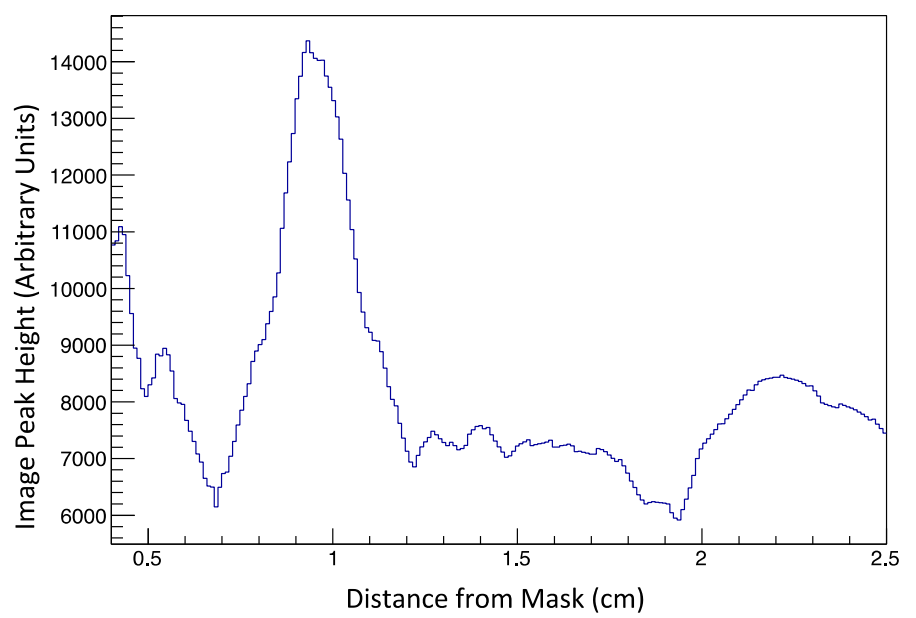

Fig. 10. Peak image value versus depth for a single event at an emulated location $9.2 \mathrm{~mm}$ from the bottom of the crystal. Sample single images for this event are shown in Fig. 9.

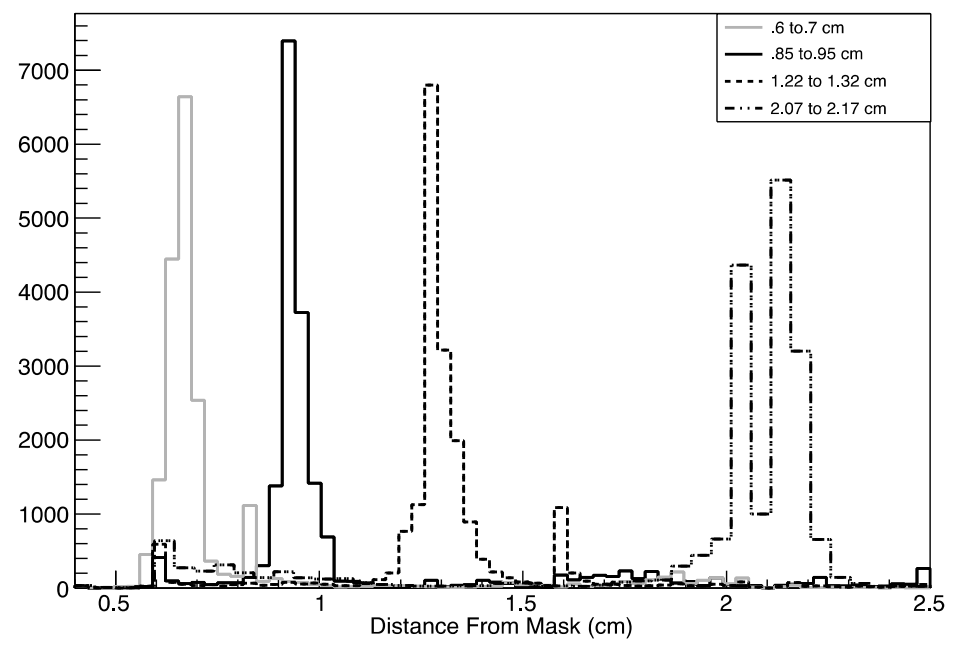

Fig. 11. Histograms of computed depths for the different emulated event depths.

The final depth results for the different emulated event depths are shown in Fig. 11. The

267 FWHM of the peaks are given in Table 1. The results include the estimate of depth based on the system geometry (Depth Resolution FWHM), the results converted to locations inside a high in-

269 dex crystal (In-crystal depth resolution) and the technique’s capabilities in-crystal after deconvo-

270 lution of the thin crystal thickness (In-crystal depth resolution, crystal thickness deconvolved). 
271 Note that for the events at the top of the crystal, the bimodal structure observed in the data is also

272 seen in light transport simulations, indicating that the structure is not just a statistical artifact.
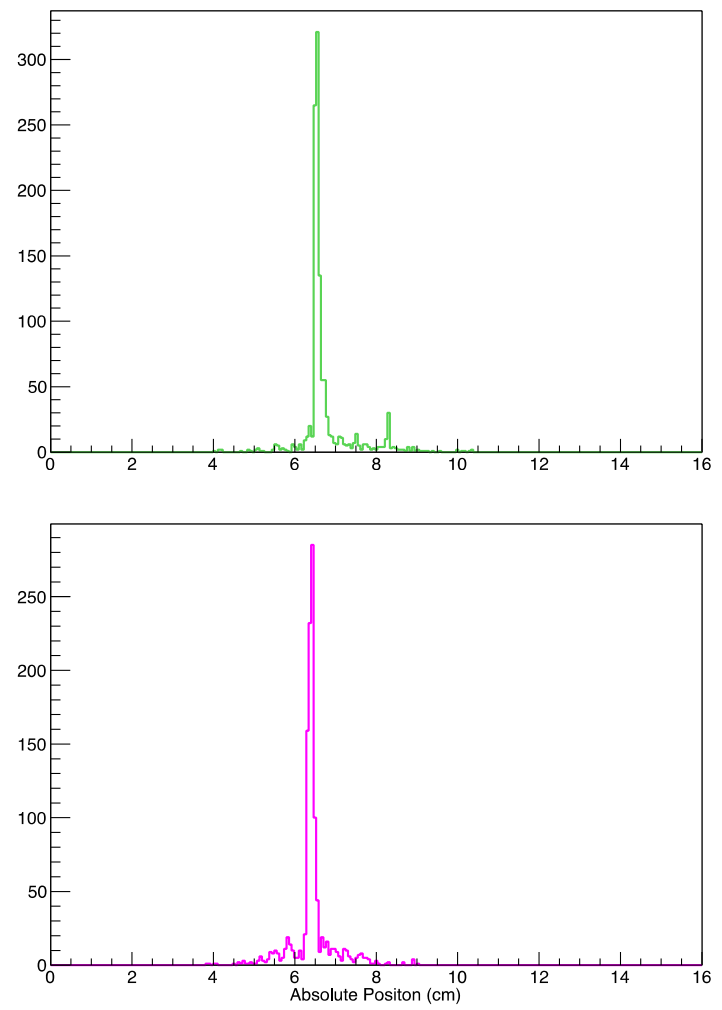

275 Fig. 12. Final $x$-distributions for event depths at the bottom (bottom) and top (top) of the crystal.

\section{$276 \quad$ 3.1.4 X-Location}

277 The final step in the event localization was to use the reconstructed image from the deter278 mined depth to correct the starting x-location to its final value. The weighted mean of the peak

279 location was determined from the image of the event to get a position finer than the pixel size of 280 the coded-aperture image at a given depth. Histograms for the X-location for all of the events at 281 each depth are given in Fig. 12.

\section{DISCUSSION}

283 The coded-aperture results clearly indicate that the technique works to obtain event locations in 284 depth. In fact, initial results appeared to be too good to be true, with the FWHM of the different 
285 peaks of Fig. 11 being narrower than the 1-mm thickness of the crystal used in the experiment.

286 Even after compensating for the 0.63 change in apparent thickness due to the difference in index

287 of refraction between the light pipe and the crystal, the peak widths were just equal to the crystal

288 thickness of $1 \mathrm{~mm} *$. This led us to wonder if the crystal might be thinner than ordered and this

289 was subsequently confirmed by a radiograph that gave a crystal thickness of $0.88 \mathrm{~mm}$. A check

290 with the crystal vendor indicated that the measured value was within their manufacturing toler-

291 ances. This indicates that the technique itself contributes little to the depth uncertainty for three

292 of the four emulated depths, and consequently the deconvolved values in Table 1 for those

293 depths are given only as an upper limit.
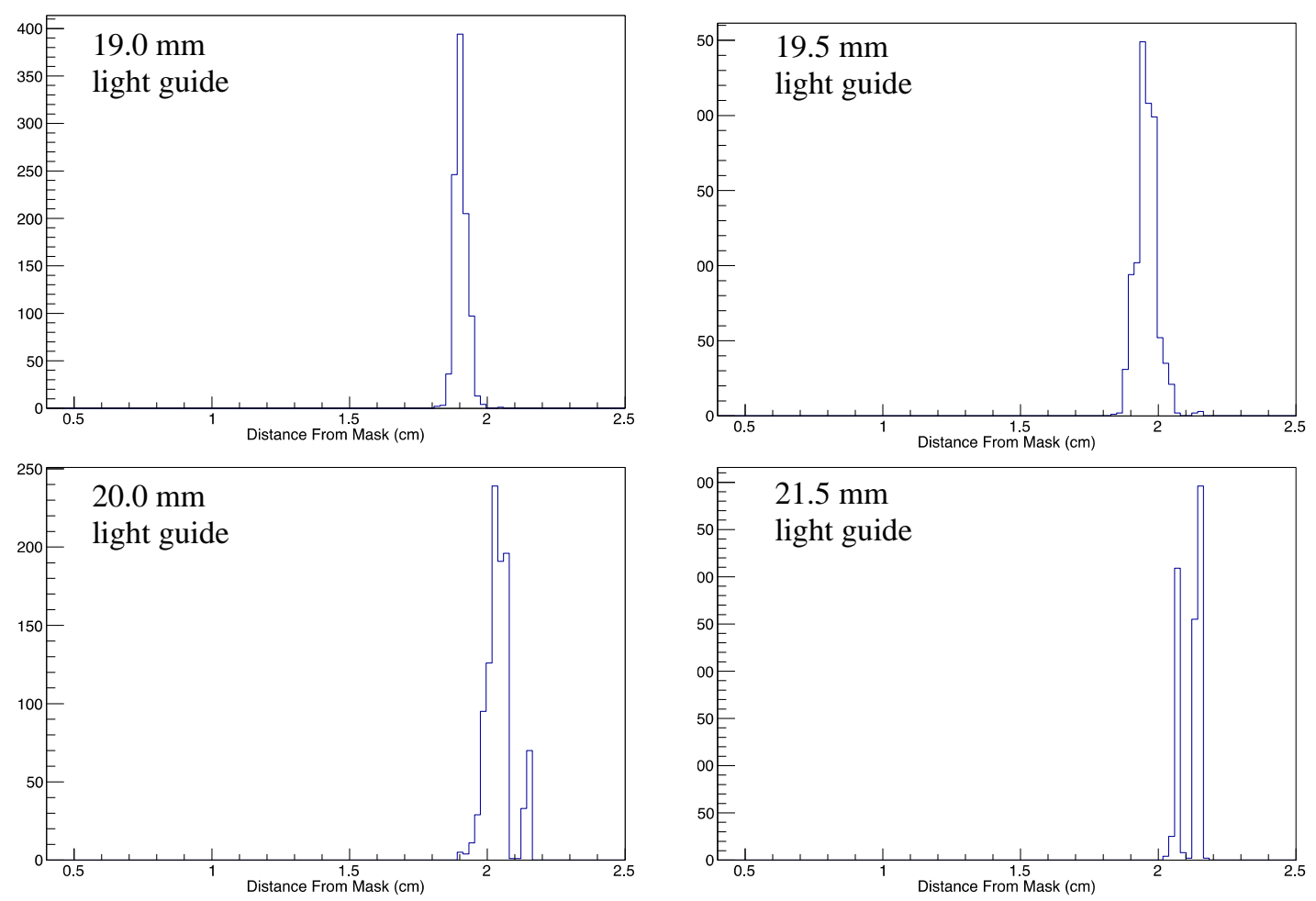

294

* Given the 356-keV of the gamma-ray events, events should occur almost uniformly throughout the depth of the thin crystal. 
Fig. 13. Simulations showing development of a bimodal structure as emulated depth increases.

Unfortunately, the uncertainty in depth for the emulated location at the top of the crystal was

297 significantly broader than $1 \mathrm{~mm}$. Simulations indicate that this is a special case in that the simu-

298 lated events show a bimodal distribution similar to that observed in the experimental data. In

299 fact, simulations performed after the measurements were completed (Fig. 13) show that the re-

300 sponse deteriorates just below the top location measured. This is likely due to a degeneracy of

301 the depth finding algorithm and indicative of the fact that the rank-seven mask may only work

302 over a finite range of depths. Better results might be obtained if a mask with more pixels was

303 used. Simulations of a fully 2D readout using the current MAPs indicate that the bimodal peak

304 structure is not an issue for a rank-19 system with a 3-cm-thick crystal. In addition, finer resolu-

305 tion readout devices are also available [16], but both the 2D system and one with a finer pitch

306 require a significant increase in the number of readout channels. Experiments with them will

307 have to wait until a more extensive data acquisition system can be assembled.

308 The performance in x-localization is equally compelling. The measured and unfolded widths

309 of the different peaks are also given in Table 1. For some of the depths, the peak width is actual-

310 ly comparable to that of the beam at the crystal, indicating that we are not resolving the error due

311 to the measurement technique. We note that the x-location was arbitrarily selected near the cen-

312 ter of the crystal and that the x-scale of the measurements is determined from the theoretical res-

313 olution of the mask and is not determined by measuring events at different locations. While

314 measurements at more x-locations would have been of value, the data acquisition system was

315 only available for a limited time and additional measurements could not be made. We note that

316 even if they had been made, the small size of the prototype would not have supported a wide

317 range of locations. This is because for the thicker emulated geometries, the light spot at the 
318 PMTs is large and the results would have included changes both due to the location above the

319 coded aperture, and the location of the event with respect to the edge of the crystal. We wanted

320 to avoid that complication. In the simulations reported earlier, a range of locations was sampled

321 and the system behaved as expected [5,6]. A full probe of performance lateral to the crystal sur-

322 face will require development of a system with a larger readout area.

\section{SUMMARY}

The results presented above indicate that the overall concept of using a shadow mask in the 325 light pipe of a scintillator-based detector to improve position resolution is sound. The perfor-

326 mance obtained with the 1D coded aperture agrees with simulations of the system, validating the 327 full 2D simulations performed earlier and indicating that this approach could, for the first time, 328 allow fine resolution of event locations in bulk scintillators.

\section{ACKNOWLEDGEMENTS}

This work was supported by the U.S. Department of Energy National Nuclear Security Administration Office of Defense Nuclear Nonproliferation Research and Development. The manu-

332 script has been authored by the Oak Ridge National Laboratory, managed by UT-Battelle LLC 333 under Contract No. DE-AC05-00OR22725 with the US Department of Energy. The US Gov-

334 ernment retains and the publisher, by accepting the article for publication, acknowledges that the 335 US Government retains a nonexclusive, paid-up, irrevocable, worldwide license to publish or 336 reproduce the published form of this manuscript, or allow others to do so, for US Government 337 purposes.

\section{REFERENCES}

339 1. H.O. Anger, Reviews of Scientific Instruments 29 (1958) 27.

340 2. M. Ricard, Reviews of Scientific Instruments 527 (2004) 124. 
341 3. J. Kataoka, et al., Nuclear Instruments and Methods in Physics Research Section A 732

342 (2013) 403.

343 4. E.E. Fenimore, T.M. Canon, Applied Optics 17 (1978) 337.

344 5. K.P. Ziock, et al, Proceedings of SPIE 8542 (2012) 854210-1-854210-12.

345 6. K.P. Ziock, M.A. Blackston, T. Van Vuure, IEEE Transactions on Nuclear Science, 60

$346 \quad$ (2013) 1390.

347 7. Hamamatsu Photonics, www.hamamatsu.com/us/en/product/category/3100/3002/

348 H9500/index.html.

349 8. S.R. Gottesman, E.E. Fenimore, Applied Optics 28, (1989) 4344.

350 9. BC-630 from Saint-Gobain Crystals, 17900 Great Lakes Parkway, Hiram, OH 44234.

351 10. S. Agostinelli, et al., Nuclear Instruments and Methods in Physics Research Section A 506

352 (2003) 250.

353 11. Strück model SIS3316-250-14, http://www.struck.de/sis3316.html.

354 12. Phillips Scientific, http://www.phillipsscientific.com/pdf/776ds.pdf.

355 13. Toppan Photomasks, 131 E. Old Settlers Blvd, Round Rock, TX 78664.

356 14. J. Braverman, M. Harrison, K.P. Ziock, Proceedings IEEE Nuclear Science Symposium, An-

357 aheim, CA, Oct. 29-Nov. 3, 335-338 (2012).

358 15. K.P. Ziock et al., Nuclear Instruments and Methods in Physics Research Section B 505

359 (2003) 420.

360 16. Photonis Planacon detector, http://www.photonis.com/en/ism/63-planacon.html. 